\section{Fatores associados à qualidade da dieta de adultos residentes na Região Metropolitana de São Paulo, Brasil, 2002}

\author{
Factors associated with dietary quality among \\ adults in Greater Metropolitan São Paulo, \\ Brazil, 2002
}

\footnotetext{
${ }^{1}$ Faculdade de Ciências Biológicas e da Saúde, Universidade Metodista de São Paulo, São Bernardo do Campo, Brasil.

2 Faculdade de Saúde Pública, Universidade de São Paulo, São Paulo, Brasil 3 Faculdade de Medicina de Botucatu, Universidade Estadual Paulista, Botucatu, Brasil.

${ }_{4}^{4}$ Faculdade de Ciências Médicas, Universidade Estadual de Campinas, Campinas, Brasil. 5 Faculdade de Medicina, Universidade de São Paulo, São Paulo, Brasil.

Correspondência J. M. Morimoto Faculdade de Ciências Biológicas e da Saúde Universidade Metodista de São Paulo.

Rua Maria Julia Capassi 110, São Bernardo do Campo, SP 09851-080, Brasil.

jmmorimoto@uol.com.br
}

\begin{abstract}
The purpose of this study was to evaluate the demographic, socioeconomic, and lifestyle factors associated with quality of diet in adults residing in Greater Metropolitan São Paulo, Brazil. This was a cross-sectional, population-based study conducted in the district of Butantã and the municipalities of Itapecerica da Serra, Embu, and Taboão da Serra, based on data from a questionnaire and 24-hour recall from 1,840 adults, both males and females, included in a household health survey (ISA-SP). Dietary quality was assessed using the Healthy Eating Index (HEI) adapted to the local reality. Linear regression analysis was used to evaluate the relationship between HEI and other variables. Most individuals (75\%) ate diets that were rated as "need improvement". The mean HEI was low for fruit, vegetables, and dairy products. Number of durable goods, head-of-family's schooling, and age $\geq 60$ years were associated with HEI in men. For women, age was associated with HEI. Energy intake was considered an adjustment variable for both genders.
\end{abstract}

Diet; Nutrition Assessment; Food Consumption; Adult

\author{
Juliana Masami Morimoto 1 \\ Maria do Rosário Dias de Oliveira Latorre ${ }^{2}$ \\ Chester Luiz Galvão César ${ }^{2}$ \\ Luana Carandina ${ }^{3}$ \\ Marilisa Berti de Azevedo Barros ${ }^{4}$ \\ Moises Goldbaum 5 \\ Regina Mara Fisberg 2
}

\section{Introdução}

Os índices de qualidade da dieta têm sido desenvolvidos para se obter uma medida resumo das principais características da dieta, facilitando a avaliação da qualidade desta em populações ou grupos de indivíduos 1 . Os mais recentes índices definem a qualidade da dieta em termos de proporcionalidade (consumir mais porções de certos grupos de alimentos e menos de outros), moderação (limitação da ingestão de alimentos e bebidas que contribuem para o consumo excessivo de gordura, colesterol, açúcares de adição, sódio e álcool) e variedade (exposição a vários componentes alimentares), tendendo a direcionar para recomendações de prevenção do desenvolvimento de doenças crônicas não transmissíveis 2,3 .

Willett 4 considera que uma ótima abordagem para análises epidemiológicas da dieta, a fim de se obter o máximo de informações sobre esta, é considerar nutrientes, alimentos e grupos de alimentos em conjunto. Com isso, os índices de qualidade da dieta (IQD) baseados em alimentos e nutrientes podem ser mais promissores, pois retêm a complexidade da dieta e permitem uma avaliação indireta de nutrientes, sem reduzir a avaliação a um único componente isolado 1 .

$\mathrm{O}$ Healthy Eating Index (HEI), desenvolvido por Kennedy et al. 5, estima a adequação e variedade da dieta, possibilitando a avaliação de possíveis mudanças nos padrões dietéticos. É cons- 
tituído por um sistema de dez componentes que caracterizam diferentes aspectos da dieta saudável: cinco grupos de alimentos (cereais, pães, tubérculos e raízes; verduras e legumes; frutas; leite e produtos lácteos; carnes, ovos e leguminosas), quatro nutrientes (níveis de ingestão de gordura total, gordura saturada, colesterol e sódio) e uma medida da variedade da dieta.

Periodicamente, o Center for Nutrition Policy and Promotion do United States Department of Agriculture utiliza o HEI com o objetivo de avaliar e monitorar a dieta dos norte-americanos. Bowman et al. 6, ao utilizarem os dados do Continuing Survey of Food Intakes by Individuals (CSFII) do período de 1994 a 1996, observaram que a maioria da população apresentava dieta que necessitava de melhora e que o consumo de frutas, leite e derivados deveria ser aumentado. As dietas com pior qualidade foram observadas em indivíduos afro-americanos, com baixa renda e menor escolaridade. Resultados semelhantes foram observados por Basiotis et al. 7 ao aplicar o HEI nos dados do National Health and Nutrition Examination Survey (NHANES) de 1999 a 2000. Os dados sugerem que, durante o intervalo de três anos entre um estudo e outro, a dieta dos norte-americanos continua "necessitando de melhora".

O IQD classifica os indivíduos em categorias de consumo alimentar, o que permite agrupá-los em segmentos semelhantes para possíveis associações com outros fatores, como sexo, idade, escolaridade, renda, consumo de bebida alcoólica e hábito de fumar. Por meio do estudo da relação entre qualidade da dieta e características sócio-econômicas, demográficas e de estilo de vida, podem ser identificados os segmentos da população que possuem risco de ter a dieta deficiente em algum nutriente ou grupo de alimentos. Conseqüentemente, torna-se possível adaptar políticas e programas de nutrição para atingir as necessidades dos segmentos da população realmente em risco. Assim, o objetivo deste estudo foi avaliar a qualidade da dieta de adultos residentes na Região Metropolitana de São Paulo, Brasil, e seus fatores associados.

\section{Materiais e método}

Este estudo foi um inquérito domiciliar populacional com delineamento transversal, realizado de 2001 a 2002. Foram estudadas duas regiões: Distrito do Butantã, no Município de São Paulo, e a região sudoeste da Grande São Paulo, constituída pelos municípios de Taboão da Serra, Embu e Itapecerica da Serra.

Para o cálculo do tamanho da amostra foram definidos quatro grupos de idade e sexo (20 a 59 anos, masculino; 20 a 59 anos, feminino; 60 anos e mais, masculino; 60 anos e mais, feminino), denominados domínios amostrais, para os quais foram garantidos números mínimos na amostra que permitissem análises posteriores em cada uma das áreas de estudo. O tamanho mínimo por domínio foi de 200 entrevistas, valor que possibilita estimar uma prevalência de 0,5 , com erro de 0,07 , considerando um alfa de 0,05 e efeito de delineamento de 2 . Considerando $20 \%$ de possíveis perdas, foram sorteados pouco mais de 250 indivíduos para cada domínio.

Utilizou-se amostragem estratificada, por conglomerados, em dois estágios. Em cada uma das áreas de pesquisa, os setores censitários foram agrupados em três estratos segundo o percentual de chefes de família com escolaridade de nível universitário. No primeiro estágio da amostragem, foram sorteados, com probabilidade proporcional ao tamanho, dez setores censitários de cada um dos três estratos, portanto trinta setores de cada área. No segundo estágio da amostragem, foram sorteados os domicílios.

Na região sudoeste da Grande São Paulo, a amostra final foi de 420 indivíduos entrevistados de 20 a 59 anos (sendo 210 homens e $210 \mathrm{mu}$ lheres) e 639 com 60 anos e mais (sendo 291 homens e 348 mulheres). Já no Distrito do Butantã, a amostra final constituiu-se de 425 adultos com 20 a 59 anos, sendo 220 homens e 205 mulheres, e 420 idosos com 60 anos e mais, sendo 201 homens e 219 mulheres. A amostra final totalizou 1.904 indivíduos adultos e idosos.

A coleta de dados foi realizada por entrevistadores previamente treinados. Dados sobre características demográficas (idade, sexo, etnia); sócio-econômicas da família (renda per capita, número de bens), do domicílio (condições de moradia e entorno) e de estilo de vida (índice de massa corporal - IMC, prática de atividade física, hábito de fumar) foram coletados por meio de aplicação de questionário. Quanto às condições da habitação classificou-se o domicílio em adequado e inadequado; habitação foi considerada adequada quando as moradias eram constituídas por casas ou apartamentos, dispondo de rede interna de água ligada à rede pública, instalação sanitária interna ligada ao sistema público de esgoto e com iluminação artificial elétrica; já habitação foi considerada inadequada quando houve ausência de uma ou mais das condições de adequação. O entorno da habitação foi dividido em adequado e inadequado, sendo considerado adequado quando havia rua pavimentada, guias e sarjetas, iluminação pública, rede pública de esgoto e coleta pública regular de lixo; já o entorno foi considerado inadequado na ausência de uma ou mais das condições de adequação. $\mathrm{O}$ 
IMC, calculado a partir das informações sobre peso (em quilogramas) e altura (em metros) referidos, foi classificado de acordo com a proposta da Organização Mundial da Saúde ${ }^{8}$. Os indivíduos foram classificados em praticantes ou não praticantes de atividade física, conforme o referido sobre este hábito. Para o hábito de fumar, foi considerado se o indivíduo era fumante, exfumante ou não fumante.

O consumo alimentar foi medido pelo método recordatório de 24 horas. O cálculo do valor nutritivo dos alimentos consumidos foi realizado utilizando-se o programa Virtual Nutri versão 1.0 (Universidade de São Paulo, São Paulo, Brasil). O banco de dados de alimentos do programa foi adaptado com a introdução de alimentos e retificações das informações da composição daqueles já contidos no programa. Para a digitação de preparações caseiras, tais como pizza, lasanha, sanduíches, optou-se pelo desdobramento destas em seus respectivos ingredientes a fim de melhor classificar os alimentos segundo os grupos da pirâmide alimentar. Para tal, foram utilizadas as padronizações de receitas propostas por $\mathrm{Pi}$ nheiro et al. 9 e Fisberg \& Villar 10. É importante salientar que a quantidade de sal de adição foi calculada e digitada separadamente para todas as preparações culinárias, tornando bastante cuidadosa a estimativa da ingestão de sal refinado. Foram excluídos da análise aqueles que consumiram menos de $500 \mathrm{kcal}$ e acima de $4.000 \mathrm{kcal}$ ( $\mathrm{n}=64)$. A amostra final foi constituída de 1.840 adultos e idosos.
Para avaliação da qualidade da dieta, foi utilizado o IQD adaptado do HEI de Kennedy et al. ${ }^{5}$ por Fisberg et al. 11. O IQD foi modificado com a inclusão do grupo das leguminosas no lugar do componente gordura saturada, pois o feijão é um alimento de consumo habitual no Brasil. Dessa forma, os seis primeiros componentes deste índice são representados pelos grupos de alimentos, três componentes são representados pelos nutrientes gordura total, colesterol e sódio, e o último, pela variedade da dieta (Tabela 1).

O IQD foi analisado como uma variável quantitativa contínua. Porém, os indivíduos também foram classificados em três categorias de acordo com Bowman et al. 6: abaixo de 51 pontos - dieta "inadequada"; entre 51 e 80 pontos - dieta que "necessita de modificação"; superior a 80 pontos - dieta "saudável".

Foi utilizada análise de regressão linear múltipla, considerando o IQD como a variável dependente e as outras variáveis (sócio-econômicas, demográficas, de estilo de vida e de caracterização do domicílio) como as independentes. As variáveis que obtiveram valor $\mathrm{p}$ menor que 0,20 na análise de regressão linear simples foram selecionadas para inclusão no modelo de regressão linear múltiplo. Para a elaboração do modelo, foi utilizada a estratégia de modelagem stepwise forward, ou seja, do modelo mais simples para o mais complexo. As variáveis que permaneceram significativas foram mantidas no modelo de regressão linear múltiplo final. A partir do modelo final de regressão elaborado para a população

Tabela 1

Descrição, variação da pontuação e critérios para pontuação máxima e mínima de cada componente do índice de qualidade da dieta (IQD).

1. Grupo dos cereais, pães, tubérculos e raízes (porções)

2. Grupo das verduras e legumes (porções)

3. Grupo das frutas (porções)

4. Grupo do leite e produtos lácteos (porções)

5. Grupo das carnes e ovos (porções)

6. Grupos das leguminosas (porções)

7. Gordura total

8. Colesterol

9. Sódio

10. Variedade da dieta

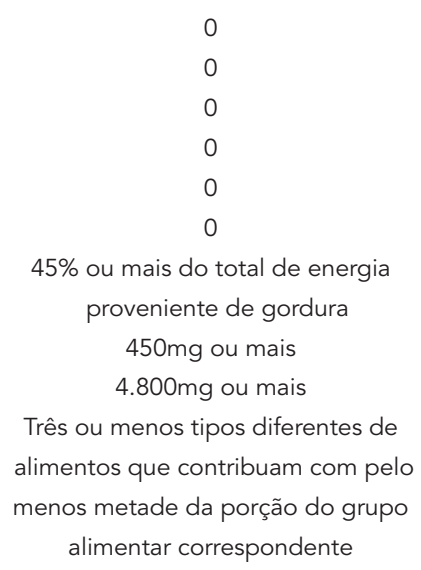

0

0

0

0

0

0

$45 \%$ ou mais do total de energia

proveniente de gordura

$450 \mathrm{mg}$ ou mais

$4.800 \mathrm{mg}$ ou mais

Três ou menos tipos diferentes de

alimentos que contribuam com pelo

menos metade da porção do grupo

alimentar correspondente

$$
\begin{gathered}
5 \text { a } 9 \\
4 \text { a } 5 \\
3 \text { a } 5 \\
3 \\
1 \text { a } 2 \\
1
\end{gathered}
$$

$30 \%$ ou menos do total de energia proveniente de gordura $300 \mathrm{mg}$ ou menos

$2.400 \mathrm{mg}$ ou menos

Oito ou mais tipos diferentes de

alimentos que contribuam com pelo

menos metade da porção do grupo alimentar correspondente 
total, estratificou-se a população por sexo - masculino e feminino - e foram estimados modelos para estas duas categorias.

Foram testadas interações entre as variáveis que permaneceram no modelo final, mas não foi constatada nenhuma interação significativa. Nenhuma variável foi considerada de controle ou confusão. Também foi realizada análise de resíduos e constatou-se que os modelos não apresentavam viés.

Na aplicação dos testes estatísticos, utilizouse o nível se significância de $5 \%$ como critério de permanência da variável no modelo final. Todas as análises consideraram as correções do desenho amostral e foram realizadas no programa Stata (Stata Corp., College Station, Estados Unidos), módulo Survey, versão 7.

O estudo foi aprovado pelo Comitê de Ética em Pesquisa da Faculdade de Saúde Pública da Universidade de São Paulo.

\section{Resultados}

A amostra deste estudo foi composta por 1.840 indivíduos adultos, que correspondem a uma população de 570.013 indivíduos residentes nas duas regiões de estudo, com idade média de 39,72 anos (erro padrão $=0,55$ ), escolaridade média de 7,3 anos (erro padrão $=0,20$ ) e renda per capita média de $\mathrm{R} \$ 490,44$ (erro padrão = 32,63).

A maioria da população de estudo era de etnia branca, sendo a proporção de homens e mulheres muito semelhante. Cerca de $40 \%$ dos indivíduos foram classificados segundo o IMC com excesso de peso (sobrepeso e obesidade) e 70\% referiram não praticar atividade física. Em relação às características sócio-econômicas, a maioria da população estudada, estava empregada, era unida ou casada (65\%), natural de fora do Estado de São Paulo (52\%), residente em casa (86\%) com condições adequadas de habitação e entorno (68\% e $64 \%$, respectivamente) (Tabela 2).

A média do IQD foi de 60,42 pontos (IC95\%: 59,33-61,50), com variação de 13,89 a 92,32 pontos. Ao classificar os indivíduos em categorias do IQD, observou-se que $4 \%$ tinham dieta saudável, $75 \%$ dieta que necessitava de modificações e $21 \%$ dieta inadequada.

Observa-se, na Tabela 3, que os valores médios de pontos dos diferentes componentes do IQD apresentaram-se baixos (abaixo de 5 pontos) para os grupos frutas, verduras e legumes, leite e produtos lácteos; valores intermediários (entre 5 e 8 pontos) para cereais, leguminosas, gordura, sódio e variedade de alimentos; e valores altos (superior a 8 pontos) para o grupo de carnes e ovos. O maior percentual de pontuações zero foi atribuído ao componente frutas (46\%). Entretanto, $81 \%$ e $73 \%$ da população estudada apresentaram pontuação máxima para os componentes carnes e ovos e colesterol, respectivamente.

$\mathrm{Na}$ análise de regressão linear simples entre o IQD e as variáveis independentes, houve correlação estatisticamente significativa para sexo, faixa etária, situação de trabalho, tipo de habitação, escolaridade do chefe da família e número de bens de consumo duráveis (Tabela 2). Ou seja, os maiores escores do IQD foram obtidos por indivíduos do sexo masculino, com idade maior ou igual a 60 anos, aposentados ou pensionistas, moradores de casa ou apartamento, com maior escolaridade e possuidores de maior número de bens de consumo duráveis.

$\mathrm{Na}$ análise múltipla, observou-se que as variáveis número de bens de consumo duráveis existentes no domicílio, escolaridade do chefe da família e idade maior ou igual a 60 anos tiveram efeitos independentes sobre o IQD. Este modelo foi estatisticamente significativo $(\mathrm{p}<0,001) \mathrm{e}$ as variáveis que compõem o modelo final explicaram a maior proporção da variância do IQD. Assim, observa-se que, quanto maior o número de bens de consumo duráveis ou maior a escolaridade do chefe da família, maior é o IQD. Em adição, estar na faixa etária de 60 anos ou mais também ocasiona um aumento médio de mais três pontos no IQD, independentemente do consumo de calorias, da escolaridade do chefe da família e do número de bens de consumo duráveis. O consumo de energia foi considerado uma variável de ajuste para o modelo de regressão final (Tabela 4).

Com base nas variáveis que permaneceram no modelo final de regressão, foram elaborados dois outros modelos de regressão múltipla estratificados por sexo, pois se observou diferença na média do IQD entre homens e mulheres, mas a variável sexo não se manteve no modelo final. Verificou-se que, para o sexo masculino, todas as variáveis inseridas se mantiveram estatisticamente significativas. Por outro lado, para o sexo feminino, idade de 60 anos ou mais foi a variável que se manteve estatisticamente significativa. Além disso, pôde-se observar que o consumo de calorias ajustou melhor o modelo de regressão linear múltiplo para mulheres do que para homens (Tabela 4).

\section{Discussão}

Este estudo foi realizado com o objetivo de avaliar os fatores demográficos, sócio-econômicos e de estilo de vida associados à qualidade da dieta de adultos residentes no Distrito do Butantã e 
Distribuição de número e porcentagem de indivíduos e média do índice de qualidade da dieta (IQD), erro padrão e valor p, segundo variáveis demográficas, de estilo de vida, sócio-econômicas e do domićílio. São Paulo, Brasil, 2001-2002.

\begin{tabular}{|c|c|c|c|c|c|}
\hline Variável & $N$ * & $\% * \star$ & Média ** & Erro padrão ** & $\mathrm{p}$ \\
\hline Sexo & & & & & 0,024 \\
\hline Masculino & 893 & 46,19 & 61,34 & 0,66 & \\
\hline Feminino & 947 & 53,81 & 59,63 & 0,65 & \\
\hline Faixa etária (anos) & & & & & 0,02 \\
\hline $20+60$ & 813 & 89,32 & 60,25 & 0,59 & \\
\hline$\geq 60$ & 1.027 & 10,68 & 61,86 & 0,46 & \\
\hline Etnia * & & & & & 0,667 \\
\hline Parda & 425 & 30,88 & 59,74 & 0,88 & \\
\hline Negra & 124 & 7,17 & 59,88 & 3,06 & \\
\hline Branca & 1.209 & 59,21 & 60,69 & 0,79 & \\
\hline Amarela & 57 & 2,28 & 62,78 & 2,52 & \\
\hline Indígena & 10 & 0,46 & 65,19 & 7,81 & \\
\hline Índice de massa corporal $\left(\mathrm{kg} / \mathrm{m}^{2}\right)$ & & & & & 0,566 \\
\hline$<18,5$ & 50 & 4,45 & 62,52 & 2,49 & \\
\hline 18,5 -25 & 772 & 55,14 & 61,08 & 0,66 & \\
\hline$\geq 25,0$ & 718 & 40,41 & 60,48 & 0,79 & \\
\hline Atividade física * & & & & & 0,333 \\
\hline Não pratica & 1.247 & 68,09 & 60,15 & 0,66 & \\
\hline Pratica & 583 & 31,91 & 61,02 & 0,70 & \\
\hline Naturalidade * & & & & & 0,989 \\
\hline Município de residência & 273 & 19,97 & 60,28 & 0,95 & \\
\hline Outro município do Estado de São Paulo & 617 & 28,29 & 60,47 & 1,06 & \\
\hline Fora do Estado de São Paulo & 941 & 51,75 & 60,45 & 0,58 & \\
\hline Situação de trabalho * & & & & & 0,028 \\
\hline Desempregado & 102 & 8,15 & 59,41 & 1,22 & \\
\hline Em atividade & 891 & 67,15 & 60,67 & 0,67 & \\
\hline Aposentado ou pensionista & 448 & 6,31 & 63,24 & 0,79 & \\
\hline Outros (dona de casa, estudante, outros) & 397 & 18,39 & 58,98 & 1,14 & \\
\hline Situação conjugal do indivíduo* & & & & & 0,813 \\
\hline Solteiro & 279 & 23,94 & 59,50 & 1,34 & \\
\hline Unido/Casado & 1.101 & 64,69 & 60,77 & 0,72 & \\
\hline Separado/Divorciado/Desquitado & 129 & 6,29 & 60,52 & 2,08 & \\
\hline Viúvo & 325 & 5,08 & 60,90 & 0,84 & \\
\hline Tipo de habitação * & & & & & 0,036 \\
\hline Barraco/Cortiço & 59 & 3,75 & 57,08 & 1,58 & \\
\hline Casa/Apartamento & 1.772 & 96,25 & 60,53 & 0,56 & \\
\hline Condições da habitação * & & & & & 0,552 \\
\hline Inadequada & 435 & 31,62 & 59,90 & 1,07 & \\
\hline Adequada & 1.366 & 68,38 & 60,78 & 0,76 & \\
\hline Condições do entorno da habitação * & & & & & 0,415 \\
\hline Inadequada & 509 & 36,21 & 59,83 & 0,85 & \\
\hline Adequada & 1.326 & 63,79 & 60,75 & 0,72 & \\
\hline Escolaridade do chefe da família (anos) & & & & & 0,004 \\
\hline 아-4 & 529 & 22,47 & 58,82 & 1,07 & \\
\hline 4 -8 & 618 & 34,54 & 59,33 & 0,69 & \\
\hline $8+-12$ & 456 & 28,27 & 62,02 & 1,14 & \\
\hline$\geq 12$ & 228 & 14,71 & 62,38 & 1,39 & \\
\hline Número de bens de consumo duráveis & & & & & 0,002 \\
\hline 아-5 & 334 & 19,41 & 58,93 & 1,29 & \\
\hline 5 卜9 & 688 & 39,05 & 59,54 & 0,76 & \\
\hline 아-12 & 509 & 26,33 & 60,46 & 0,99 & \\
\hline$\geq 12$ & 303 & 15,21 & 64,59 & 1,16 & \\
\hline
\end{tabular}

* Excluídos os indivíduos com informação ignorada;

** Valores ponderados conforme desenho amostral. 
Estatística descritiva para os escores de cada componente do índice de qualidade da dieta (IQD) e percentual de indivíduos que obtiveram pontuação mínima (0) ou máxima (10). São Paulo, Brasil, 2001-2002.

\begin{tabular}{|c|c|c|c|c|c|}
\hline Componente do IQD & Mediana * (pontos) & Média * (pontos) & IC95\% * & Escore $0(\%)$ * & Escore $10(\%)$ * \\
\hline Cereais, pães, tubérculos e raízes & 7,00 & 6,84 & $6,60-7,08$ & 0,21 & 25,11 \\
\hline Verduras e legumes & 4,47 & 4,77 & $4,52-5,02$ & 3,96 & 13,43 \\
\hline Frutas & 0,21 & 3,71 & $3,37-4,05$ & 45,90 & 22,55 \\
\hline Leite e derivados & 2,22 & 2,90 & $2,67-3,12$ & 25,33 & 4,69 \\
\hline Carnes e ovos & 10,00 & 8,94 & $8,76-9,12$ & 4,70 & 81,15 \\
\hline Leguminosas & 9,07 & 6,38 & $5,98-6,79$ & 27,64 & 43,72 \\
\hline Gordura total & 5,70 & 5,51 & $5,17-5,84$ & 17,03 & 27,22 \\
\hline Colesterol & 10,00 & 8,17 & $7,93-8,41$ & 11,49 & 72,85 \\
\hline Sódio & 7,86 & 6,39 & $6,11-6,68$ & 14,82 & 34,81 \\
\hline Variedade de alimentos & 8,00 & 6,82 & $6,55-7,09$ & 5,96 & 39,07 \\
\hline
\end{tabular}

* Valores ponderados conforme desenho amostral.

Tabela 4

Resultado da análise de regressão múltipla final estratificada por sexo. São Paulo, Brasil, 2001-2002.

\begin{tabular}{|c|c|c|c|c|c|c|}
\hline \multirow[t]{2}{*}{ Variáveis independentes } & \multicolumn{2}{|c|}{$\begin{array}{l}\text { Sexo masculino } \\
\qquad(n=888)\end{array}$} & \multicolumn{2}{|c|}{$\begin{array}{c}\text { Sexo feminino } \\
(n=939)\end{array}$} & \multicolumn{2}{|c|}{$\begin{array}{c}\text { Total } \\
(\mathrm{n}=1.827)\end{array}$} \\
\hline & $\beta$ * & $\mathbf{p}$ & $\beta$ * & $\mathrm{p}$ & $\beta$ * & $\mathbf{p}$ \\
\hline Ingestão de energia & 0,002 & 0,018 & 0,004 & $<0,001$ & 0,003 & $<0,001$ \\
\hline Número de bens de consumo duráveis & 0,276 & 0,032 & 0,449 & 0,092 & 0,365 & 0,018 \\
\hline Escolaridade do chefe em anos & 0,465 & 0,007 & 0,057 & 0,760 & 0,258 & 0,044 \\
\hline Faixa etária de 60 anos ou mais ** & 2,564 & 0,005 & 3,077 & 0,003 & 2,990 & $<0,001$ \\
\hline$r^{2}$ & \multicolumn{2}{|c|}{0,060} & \multicolumn{2}{|c|}{0,081} & \multicolumn{2}{|c|}{0,068} \\
\hline p do modelo & \multicolumn{2}{|c|}{$<0,001$} & \multicolumn{2}{|c|}{$<0,001$} & \multicolumn{2}{|c|}{$<0,001$} \\
\hline
\end{tabular}

* Coeficiente de regressão:

** Em relação à faixa etária de 20 a 59 anos.

nos municípios de Taboão da Serra, Embu e Itapecerica da Serra, áreas que compõem a Região Metropolitana de São Paulo.

Vale ressaltar que uma limitação deste estudo foi a avaliação do consumo alimentar de apenas um dia, já que as dietas dos indivíduos podem variar de dia para dia, e a medida de um único dia não representa a ingestão habitual do indivíduo. Porém, o recordatório de 24 horas fornece informações detalhadas sobre os tipos de alimentos consumidos e suas quantidades, o que torna útil o seu emprego em inquéritos populacionais, como o do presente estudo. Segundo Willett 4, a aplicação de um único recordatório de 24 horas pode ser adequada para estimar valores médios de ingestão no grupo, desde que o tamanho da amostra seja adequado para este propósito. Este fato é observado no Third National Health and Nutrition Examination Survey (NHANES III), estudo com amostra representativa da população norte-americana, que utilizou como metodologia de inquérito alimentar um recordatório de 24 horas e tem utilizado o HEI para avaliar a qualidade da dieta dos indivíduos observados 5,12,13.

A média do IQD foi de 60,42 pontos no presente estudo, indicando qualidade moderada da dieta. Rafferty et al. 14, utilizando o Michigan Healthy Diet Indicator (MI-HDI), que é um índice de qualidade da dieta baseado no HEI, obtiveram média inferior ao observado no presente estudo (55,7 pontos) entre indivíduos de 18 anos e mais. Entretanto, outros estudos que utilizaram o HEI obtiveram resultados superiores, como Kennedy et al. ${ }^{5}$, que pela primeira vez aplicaram o HEI 
em amostra representativa da população norteamericana, observando média de 63,9 pontos, e Bowman et al. 6, que, ao utilizarem dados do CSFII de 1994 a 1996, obtiveram média de 63,6 pontos. Essa média permaneceu praticamente inalterada (63,8 pontos) quando o HEI foi novamente aplicado em dados norte-americanos mais recentes do NHANES, de 1999 a 2000 7. Ao aplicar o HEI nos dados do NHANES III, Guo et al. 12 encontraram média de 63,2 pontos entre adultos de 20 a 75 anos. McCullough et al. 15,16 observaram valores médios do HEI de 64,4 pontos em mulheres e 68,3 pontos em homens, também pontuações superiores às encontradas neste estudo. Os valores médios dos índices de qualidade da dieta dos estudos citados anteriormente se encontram entre 50 e 70 pontos, o que pode significar que, em média, a qualidade da dieta das pessoas necessita de modificações.

O percentual de indivíduos com dieta saudável observado no presente estudo (4\%) foi inferior ao observado na literatura. Utilizando dados populacionais norte-americanos, Bowman et al. 6 encontraram percentual de indivíduos com dieta saudável três vezes superior (12\%); Basiotis et al. 7 e Guo et al. 12 obtiveram resultados cerca de duas vezes maior (10\%) do que o obtido neste estudo.

Ao comparar as pontuações médias dos componentes do IQD obtidas nesse estudo com os dados da literatura, observou-se que houve semelhança com os escores dos componentes frutas, colesterol e variedade de alimentos 6,7,14. Bowman et al. 6 e Basiotis et al. ${ }^{7}$ obtiveram médias superiores para os componentes verduras e legumes (6,0 pontos em ambos), leite e produtos lácteos (5,4 e 5,9 pontos, respectivamente) e gordura total (6,9 pontos em ambos). Os resultados de Rafferty et al. 14 foram superiores para o componente leite e produtos lácteos (6,7 pontos) e gordura total ( 7,3 pontos) e inferiores para cereais (2,5 pontos) e verduras e legumes ( 3,8 pontos). As médias de pontos do componente carnes e ovos não puderam ser comparadas aos dados da literatura, pois, neste estudo, as leguminosas fizeram parte de um componente em separado, diferentemente do HEI original.

A média de pontos do componente fruta foi semelhante aos resultados de estudos encontrados na literatura 5,6,7,14. Além de a pontuação média para o consumo de frutas ter sido baixa ( 3,7 pontos), observa-se que $50 \%$ dos indivíduos deste estudo obtiveram pontuação próxima de zero, o que significa um consumo de praticamente nenhuma porção de fruta, em um dia, por metade da população de estudo. A importância do consumo adequado de frutas na prevenção de doenças já está amplamente divulgada na literatura 17,18,19,20,21.
No presente estudo, nota-se também consumo muito baixo de leite e produtos lácteos, já que a pontuação média alcançada equivale ao consumo de menos de uma porção deste grupo alimentar por dia, resultado bem inferior ao observado em outros estudos 19,22. É interessante destacar que a recomendação de consumo de leite e produtos lácteos é de três porções por dia, o que equivale a aproximadamente três copos de requeijão de leite integral que fornecem cerca de 800mg de cálcio. Essa quantidade é inferior à recomendação de ingestão adequada (adequate intake) estabelecida para cálcio, que varia de $1.000 \mathrm{mg}-19$ a 50 anos - a 1.200mg - 51 anos e mais 23. No Estudo Multicêntrico sobre Consumo Alimentar realizado em cinco municípios brasileiros (Campinas - São Paulo, Goiânia - Goiás, Ouro Preto - Minas Gerais, Curitiba - Paraná e Rio de Janeiro), já havia sido observada uma inadequação da disponibilidade de cálcio para todas as faixas de renda e idade, com exceção do Rio de Janeiro que apresentou adequação de $100 \%$ para a disponibilidade de cálcio na faixa de maior renda 24 .

Os resultados do presente estudo demonstraram que a qualidade da dieta variou significativamente de acordo com as características demográficas de sexo e faixa etária. Esses resultados são semelhantes aos observados na literatura 6,7 , os quais verificaram média do HEI maior entre mulheres do que entre homens e maior nos indivíduos com idade superior a 50 anos do que nos adultos com idade inferior. Guo et al. 12, analisando os dados do NHANES III, encontraram que indivíduos do sexo feminino e com mais de 60 anos têm média do HEI maior do que os adultos do sexo masculino e com idade inferior a 60 anos, respectivamente. Estudo que aplicou o MI-HDI (índice de qualidade da dieta baseado no HEI) em dados do Michigan Behavioral Risk Factor Surveillance System observou que a média deste índice foi estatisticamente maior entre mulheres ( 58,8 pontos) do que entre homens (52,3 pontos) e aumentou de acordo com a idade 14 . Forshee \& Storey 25 , ao analisarem dados do CSFII 1994-1996, 1998, por meio do HEI, observaram que a dieta dos homens apresentava menor qualidade do que a das mulheres. A influência da idade sobre a qualidade da dieta pode refletir uma mudança de consciência sobre a saúde com o aumento da idade, interferindo diretamente nas escolhas alimentares pelo grupo de maior idade, ou na manutenção dos hábitos em face da menor exposição ao ambiente. Deste modo, as políticas de promoção de estilo de vida saudável deveriam ser implementadas o mais precocemente possível na população. 
Guo et al. 12 encontraram diferenças nas médias de HEI segundo características de estilo de vida. Indivíduos que relataram praticar exercício três ou mais vezes por semana tiveram média maior do que os que não praticavam nada ou que o faziam até três vezes por semana. Observou-se também que fumantes tiveram média do índice menor que não fumantes (58,6 e 65,2 pontos, respectivamente). Ao contrário do verificado na literatura, as associações entre o IQD e atividade física e hábito de fumar não foram observadas neste estudo.

Em relação às características sócio-econômicas, os resultados do presente estudo demonstraram que a qualidade da dieta melhora de acordo com o aumento do nível de escolaridade, tanto do indivíduo quanto do chefe da família, e de condição sócio-econômica (número de bens de consumo e renda per capita). Resultados semelhantes foram relatados por outros estudos 6,7,12, que observaram o aumento do HEI de acordo com os níveis de escolaridade e renda. Rafferty et al. 14 encontraram que o índice de qualidade da dieta MI-HDI obteve média maior entre os indivíduos com nível superior completo do que entre os com menor escolaridade, mas não observou diferenças estatisticamente significativas entre as categorias de renda familiar. Thiele et al. 26 também verificaram que mulheres com alto nível de escolaridade e homens com escolaridade intermediária apresentavam escores elevados do IQD. Utilizando dados do CSFII e Diet and Health Knowledge Survey de indivíduos adultos, PérezEscamilla \& Haldeman 27 também observaram que a educação formal estava positivamente associada à qualidade da dieta, medida pelo HEI. Forshee \& Storey 25 observaram que a renda familiar teve associação positiva com o HEI. A variável renda não se manteve no modelo final.

Segundo Popkin et al. 28 , o mecanismo pelo qual o maior nível de escolaridade promove dietas saudáveis não foi completamente elucidado, mas pode ser causado pela difusão de conhecimento, experiências, atitudes e crenças que ocorre enquanto as pessoas estão no sistema educacional. Assim, indivíduos com escolaridade mais elevada tendem a apresentar maior conhecimento para aquisição de alimentos, aumentando a variedade da dieta através do consumo de, principalmente, mais frutas e hortaliças e, conseqüentemente, aumentando a pontuação do IQD. O impacto da escolaridade do chefe da família sobre a capacidade de escolha dos alimentos foi encontrado por Fisberg et al. 29 em estudo que utilizou os dados de consumo alimentar do Inquérito de Saúde do Estado de São Paulo (ISA-SP), o que incluía parte da região da Grande São Paulo, Distrito do Butantã e os municípios de Campinas e Botucatu. Observouse o aumento das médias de escores de verduras e legumes, frutas, leite e produtos lácteos e variedade de alimentos conforme aumento da escolaridade do chefe da família. Para o grupo das leguminosas, foi encontrada situação inversa: a categoria de maior escolaridade do chefe da família apresentava a menor média de pontos para esse componente. Provavelmente, na população de menor renda, o hábito alimentar tradicional brasileiro composto por arroz e feijão esteja mantido devido ao fácil acesso a estes alimentos (boa disponibilidade e baixo custo). A combinação de arroz e feijão é benéfica ao organismo principalmente quanto ao fornecimento dos aminoácidos essenciais.

Durante a modelagem múltipla, observouse que a variável escolaridade do chefe da família teve um efeito importante sobre o IQD, podendo assim ser considerada uma variável indicadora da condição sócio-econômica do indivíduo ou da família. O uso deste indicador tem se destacado devido à facilidade de coleta e relativa precisão, o que permite uma classificação satisfatória das condições de vida de populações. César 30, ao analisar as condições de vida do estudo ISA-SP, verificou que o grau de escolaridade do chefe de família mostrou-se um bom indicador da condição de vida descrita em suas diferentes dimensões.

Após estratificação do modelo final de regressão por sexo, apenas o consumo de calorias e idade de 60 anos ou mais foram as variáveis que se mantiveram estatisticamente significativas paras as mulheres. Para os homens, todas as variáveis do modelo múltiplo final mantiveram sua significância estatística. A escolaridade do chefe da família manteve efeito significativo sobre o IQD de homens, porque aproximadamente $70 \%$ dos chefes de família da população de estudo eram do sexo masculino. Esta proporção é consistente com a Pesquisa Nacional por Amostra de Domicílios (PNAD), na qual $67 \%$ da população de referência da Região Metropolitana de São Paulo era do sexo masculino ${ }^{31}$.

É interessante observar que o efeito do consumo de calorias e da idade é maior em mulheres do que em homens, já que ter 60 anos ou mais impõe um efeito 0,5 ponto a mais nas mulheres do que nos homens, e o efeito do ajuste do consumo de calorias também foi maior entre as mulheres.

\section{Conclusão}

Os resultados deste estudo sugerem que condição sócio-econômica (representada pela esco- 
laridade do chefe da família e pelo número de bens de consumo duráveis) e faixa etária são fatores que podem ser utilizados para direcionar os esforços em atividades de promoção da saúde e de uma alimentação saudável. São necessárias políticas públicas em alimentação e nutrição para atingir toda a população, mas principalmente direcionadas para adultos jovens, de menor renda e residentes em famílias com chefes de baixa escolaridade. Deve-se ter atenção especial na orientação sobre o aumento do consumo de frutas, hortaliças, leite e produtos lácteos. Campanhas educativas de incentivo para a elaboração de hortas comunitárias e aumento do consumo de alimentos da estação, que geralmente têm custo mais baixo, são algumas ações que podem ser realizadas para melhorar a disponibilidade de alimentos para toda a população, permitindo que as pessoas coloquem as orientações obtidas sobre dieta saudável em prática.

\section{Resumo}

Objetivou-se avaliar os fatores demográficos, sócioeconômicos e de estilo de vida associados à qualidade da dieta de adultos residentes na Região Metropolitana de São Paulo, Brasil. Estudo transversal, por meio de inquérito domiciliar, de base populacional, foi realizado no Distrito do Butantã e nos municípios de Itapecerica da Serra, Embu e Taboão da Serra. Utilizaram-se dados de um questionário e um recordatório de 24 horas de 1.840 adultos de 20 anos ou mais, de ambos os sexos, incluídos em um inquérito de saúde (ISA-SP). A qualidade da dieta foi avaliada através do indice de qualidade da dieta (IQD) adaptado para a realidade local. Utilizou-se análise de regressão linear para avaliar a associação entre o IQD e as demais variáveis. A maioria da população (75\%) apresentou dieta que necessita de melhora. Observaram-se médias baixas para os componentes: frutas, verduras e legumes, leite e derivados. Número de bens de consumo duráveis, escolaridade do chefe da família e ter 60 anos ou mais se associaram ao IQD em homens. Para as mulheres, a faixa etária se associou ao IQD. Em ambos os modelos, o consumo de calorias se manteve como variável de ajuste.

Dieta; Avaliação Nutricional; Consumo Alimentar; Adulto

\section{Colaboradores}

J. M. Morimoto participou da análise, interpretação e discussão dos resultados, redação e revisão do texto. M. R. D. O. Latorre colaborou nas análises e interpretação dos resultados. C. L. G. César coordenou a pesquisa principal, o planejamento, delineamento e coleta de dados. L. Carandina, M. B. A. Barros e M. Goldbaum participaram da coordenação da pesquisa, do planejamento e delineamento do estudo principal. R. M. Fisberg contribuiu na coordenação da pesquisa principal, colaborou na análise, interpretação, discussão e redação dos resultados e na redação do texto.

\section{Agradecimentos}

O presente trabalho foi realizado com apoio do Conselho Nacional de Desenvolvimento Científico e Tecnológico (CNPq). 


\section{Referências}

1. Kant AK. Indexes of overall diet quality: a review. J Am Diet Assoc 1996; 96:785-91.

2. Haines PS, Siega-Riz AM, Popkin BM. The diet quality index revised: a measurement instrument for populations. J Am Diet Assoc 1999; 99:697-704.

3. Lee RD, Nieman DC. Standards for nutrient intake. In: Lee RD, Nieman DC, editors. Nutritional assessment. 3rd Ed. New York: McGraw-Hill; 2003. p. 12-72.

4. Willett WC. Nutritional epidemiology. 2nd Ed. New York: Oxford University Press; 1998

5. Kennedy ET, Ohls J, Carlson S, Fleming K. The healthy eating index: design and applications. J Am Diet Assoc 1995; 95:1103-8.

6. Bowman SA, Lino M, Gerrior SA, Basiotis PP. The healthy eating index: 1994-96. Washington DC: Center for Nutrition Policy and Promotion, U.S. Department of Agriculture; 1998.

7. Basiotis PP, Carlson A, Gerrior SA, Juan WY, Lino M. The healthy eating index: 1999-2000. Washington DC: Center for Nutrition Policy and Promotion, U.S. Department of Agriculture; 2002.

8. World Health Organization. Report of a WHO Consultation on Obesity. Obesity: preventing and managing the global epidemic. Geneva: World Health Organization; 2000. (WHO Technical Report Series, 894).

9. Pinheiro ABV, Lacerda EMA, Benzecry EH, Gomes MCS, Costa VM. Tabela para avaliação de consumo alimentar em medidas caseiras. 4ạ Ed. São Paulo: Editora Atheneu; 2000.

10. Fisberg RM, Villar BS. Manual de receitas e medidas caseiras para cálculo de inquéritos alimentares: manual elaborado para auxiliar o processamento de inquéritos alimentares. São Paulo: Signus Editora; 2002.

11. Fisberg RM, Slater B, Barros RR, Lima FD, Carandina L, Barros MBA, et al. Índice de qualidade da dieta: avaliação da adaptação e aplicabilidade. Rev Nutr PUCCAMP 2004; 17:301-8.

12. Guo X, Warden BA, Paeratakul S, Bray GA. Healthy eating index and obesity. Eur J Clin Nutr 2004; 58:1580-6.

13. Ford ES, Mokdad AH, Liu S. Healthy eating index and C-reative protein concentration: findings from the National Health and Nutrition Examination Survey III, 1988-1994. Eur J Clin Nutr 2005; 59:27883.

14. Rafferty AP, Anderson JV, McGee HB, Miller CE. A healthy diet indicator: quantifying compliance with the dietary guidelines using the BRFSS. Prev Med 2002; 35:9-15.

15. McCullough ML, Feskanich D, Rimm EB, Giovannucci EL, Ascherio A, Variyam JN, et al. Adherence to the dietary guidelines for Americans and risk of major chronic disease in men. Am J Clin Nutr 2000; 72:1223-31

16. McCullough ML, Feskanich D, Stampfer MJ, Rosner BA, Hu FB, Hunter DJ, et al. Adherence to the dietary guidelines for Americans and risk of major chronic disease in women. Am J Clin Nutr 2000; 72:1214-22.
17. Slattery ML, Boucher KM, Caan BJ, Potter JD, Ma KN. Eating patterns and risk of colon cancer. Am J Epidemiol 1998; 148:4-16.

18. Marchioni DML, Fisberg RM, Góis Filho JF, Kowalsky LP, Carvalho MB, Abrahão M, et al. Fatores dietéticos e câncer oral: um estudo caso-controle na Região Metropolitana de São Paulo, Brasil. Cad Saúde Pública 2007; 23:553-64.

19. Joint WHO/FAO Expert Consultation on Diet, Nutrition and the Prevention of Chronic Diseases, World Health Organization. Diet, nutrition and the prevention of chronic diseases. Geneva: World Health Organization; 2003. (WHO Technical Report Series, 916).

20. Hu FB, Willett WC. Optimal diets for prevention of coronary heart disease. JAMA 2002; 288:2569-78.

21. Ness AR, Powles JW. Fruit and vegetables, and cardiovascular disease: a review. Int J Epidemiol 1997; 26:1-13.

22. Prentice A. Diet nutrition and the prevention of osteoporosis. Public Health Nutr 2004; 7:227-43.

23. Institute of Medicine. Dietary reference intakes: applications in dietary assessment. Washington DC: National Academies Press; 2000.

24. Galeazzi MAM, Domene SMA, Sichieri R, organizadores. Estudo multicêntrico sobre consumo alimentar. Cadernos de Debate 1997; no. espec.

25. Forshee RA, Storey ML. Demographics, not beverage consumption, is associated with diet quality. Int J Food Sci Nutr 2006; 57:494-511.

26. Thiele S, Mensink GBM, Beitz R. Determinants of diet quality. Public Health Nutr 2004; 7:29-37.

27. Pérez-Escamilla R, Haldeman L. Food label use modifies association of income with dietary quality. J Nutr 2002; 132:768-72.

28. Popkin BM, Zizza C, Siega-Riz AM. Who is leading the change? U.S. dietary quality comparison between 1965 and 1996. Am J Prev Med 2003; 25:1-8.

29. Fisberg RM, Slater B, Morimoto JM, Bueno MB. Hábito alimentar: qualidade da dieta. In: César CLG, Carandina L, Alves MCGP, Barros MBA, Goldbaum M, organizadores. Saúde e condição de vida em São Paulo: inquérito multicêntrico de saúde no Estado de São Paulo. São Paulo: Faculdade de Saúde Pública, Universidade de São Paulo; 2005. p. 81-9.

30. Cesar CLG. Condição de vida. In: César CLG, Carandina L, Alves MCGP, Barros MBA, Goldbaum M, organizadores. Saúde e condição de vida em São Paulo: inquérito multicêntrico de saúde no Estado de São Paulo. São Paulo: Faculdade de Saúde Pública, Universidade de São Paulo; 2005. p. 63-78.

31. Instituto Brasileiro de Geografia e Estatística. Síntese de indicadores sociais 2003. Rio de Janeiro: Instituto Brasileiro de Geografia e Estatística; 2004. (Estudos \& Pesquisas. Informação Demográfica e Socioeconômica, 12).

Recebido em 09/Out/2006

Versão final reapresentada em 15/Mai/2007 Aprovado em 28/Mai/2007 